\title{
Development of a simple MR-compatible vibrotactile stimulator using a planar-coil-type actuator
}

\author{
Hyung-Sik Kim • Mi-Hyun Choi • Yoon-Gi Chung • \\ Sung-Phil Kim • Jae-Hoon Jun • Jang-Yeon Park • \\ Jeong-Han Yi • Jong-Rak Park • Dae-Woon Lim • \\ Soon-Cheol Chung
}

Published online: 6 October 2012

(C) Psychonomic Society, Inc. 2012 advantages of providing highly repeatable stimulation, being durable, being able to assume various shapes, and having an adjustable contact area with the skin. The new stimulator operated stably in an MR environment without affecting the MR images. Using functional magnetic resonance imaging, we observed the brain activation changes resulting from stimulation frequency and intensity changes.

Keywords MR-compatible · Planar-coil-type actuator · Vibrotactile stimulator $\cdot$ Electromagnetic

Many studies related to tactile perception and cognition have been performed using functional magnetic resonance imaging (fMRI; Anton et al., 1996; Bleton et al., 2011; Geyer et al., 2001; Roiha et al., 2011; Tsekos, Khanicheh, Christoforou, \& Mavroidis, 2007). To perform human tactile studies in magnetic resonance (MR) circumstances, the stimulators should not be affected by the MR scanner, so

Y.-G. Chung $\cdot$ S.-P. Kim

Department of Brain and Cognitive Engineering, Korea

University,

Seoul, South Korea

Y.-G. Chung

e-mail: uskeywest@korea.ac.kr

S.-P. Kim

e-mail: spkim@korea.ac.kr

J.-R. Park

Department of Photonic Engineering, Chosun University,

Gwangju, South Korea

e-mail: ejrpark@chosun.ac.kr

D.-W. Lim

Department of Information \& Communication Engineering,

Dongguk University,

Seoul, South Korea

e-mail: dwlim01@dgu.edu 
as not to produce MR image distortions. The frequency, intensity, time, and area of stimulation can change the magnitude, region, and pattern of neural activation (Lynette \& Nadine, 2008). Thus, the development of an MR-compatible stimulator that can quantitatively control stimulation variables is necessary for successful tactile stimulation studies.

Until now, vibrotactile stimulators have been mainly used for fMRI studies (Chakravarty, Broadbent, Rosa-Neto, Lambert, \& Collins, 2009). For vibrotactile stimulation in MR fields, pneumatic (Briggs et al., 2004; Chakravarty et al., 2009; Gallasch et al., 2010; Montant, Romaiquere, \& Roll, 2009; Wienbruch, Candia, Svensson, Kleiser, \& Kollias, 2006), piezoelectric-element (Gizewski et al., 2005; Harrington, Wright, \& Downs, 2000), shaft (Golaszewski et al., 2002), and electromagnetic (Graham, Staines, Nelson, Plewes, \& McIlroy, 2001) methods have been used.

The pneumatic method can cause normal indentation by air injection on the skin (Briggs et al., 2004; Wienbruch et al., 2006) and can cause vibrotactile stimulation by air flow control (Chakravarty et al., 2009; Montant et al., 2009) or air bag expansion (Gallasch et al., 2010). However, these methods have low spatial resolution, limited stimulation frequencies $(<100 \mathrm{~Hz})$, poor intensity control, and long transient response times.

Piezoelectric-element methods have a wide frequency range, but their stimulation intensity is small and varies with frequency changes, and the stimulator is difficult to attach to the target (Harrington et al., 2000). Also, the structure of the high-voltage hardware needed to operate piezoelectric elements is complicated. Recently, a high-intensity stimulator has been developed, but it is limited to a single channel, and the actuator has a complicated structure (Gizewski et al., 2005).

The shaft method uses a DC motor and shaft to cause vibrating stimulation by changing the turning force. The stimulation intensity is large, but the stimulation frequency is below $130 \mathrm{~Hz}$ and the system is bulky. Also, the stimulator is complicated and large, with a limited stimulation area (e.g., the palm; Golaszewski et al., 2002).

The electromagnetic method can cause vibrating stimulation using the strong and homogeneous static magnetic field of the MR scanner. The conventional system has limited stimulation frequency, intensity, and time, with inconsistent stimulation intensity that varies according to the position and orientation of the actuator. Since this method is used with a winding coil, the actuator is large and not expandable to multichannel stimuli (Graham et al., 2001). However, as compared to other methods of vibrotactile stimulation in MR field, this method uses low voltage, and the system is simple and small, with low cost.

The objective of this study was to develop a simple MRcompatible vibrotactile stimulator that would capitalize on the advantages of the electromagnetic method and overcome the disadvantages of conventional tactile stimulators (e.g., limited stimulation intensity, frequency, time, and channel, with a bulky and complicated actuator). The system that we developed uses a microcontroller for precise control of the stimulation parameters, and planar technology to make a high-quality actuator.

\section{Development of the MR-compatible vibrotactile stimulator using a planar-coil-type actuator}

Figure 1 shows the schematic of the vibrotactile stimulator. This stimulator consists of a control unit, a drive unit, and an actuator. The control unit is positioned outside, and the drive unit and actuator inside, the MR shield room.

The control unit controls stimulation parameters such as stimulation frequency, intensity, time, and channels. It generates sinusoidal stimulation signals and performs synchronization with the MR system. The main controller uses an AVR-family ATMEGA128 controller (Atmel, San Jose, CA), which is a general purpose 8-bit microcontroller (Fig. 2). This controller works at approximately a 4.5- to $5.5-\mathrm{V}$ operating voltage, and it has several capabilities, such as an eight-channel, 10-bit analog-to-digital converter (ADC) and 8- and 16-bit timer/counters.

Those stimulation parameters can be controlled with EPrime software (Psychology Software Tools Inc., Sharpsburg, PA) by a PC. E-Prime is well-known software that is used as a visual and auditory stimulation tool in psychology, neuroscience, and the cognitive and behavioral sciences (Gallace, Tan, Haggard, \& Spence, 2007; Ho \& Spence, 2007). Therefore, tactile, auditory, and visual stimulation can be generated simultaneously using the software (Kim et al., 2011). The stimulation parameters and signals are delivered to the control unit through the LPT-1 port in a PC. The user can also input stimulation parameters using buttons on the control unit and can monitor performance on a liquid crystal display (LCD). The dimensions of the control unit are $120 \times 90 \times$ $1.5 \mathrm{~mm}^{3}(\mathrm{~W} \times \mathrm{L} \times \mathrm{H})$, and the weight is $160 \mathrm{~g}$.

The output of the stimulation signal is a sinusoidal wave. MATLAB (MathWorks, Natick, MA) is used to generate the binary code for a sinusoidal wave and to save a look-up table in the microcontroller. Binary codes can be generated with 12-bit digital-to-analog converter (DAC) AD7545A (Analog Devices, Norwood, MA) and the timer/counter function of the microcontroller can control the stimulation frequency $(20 \sim 400 \mathrm{~Hz}$, at 40 levels) of the signals. A digital potentiometer (Analog Devices Model AD5290; R1 in Fig. 2), which is controlled by the serial peripheral interface (SPI) function of the microcontroller, can control the stimulation intensity $(0 \sim 7 \mathrm{~V}$, at 256 levels), time (from microseconds to seconds), and channels (one to three). Electric signals can be transformed to optic signals by use of a light-emitting diode (LED), and stimulation optic 


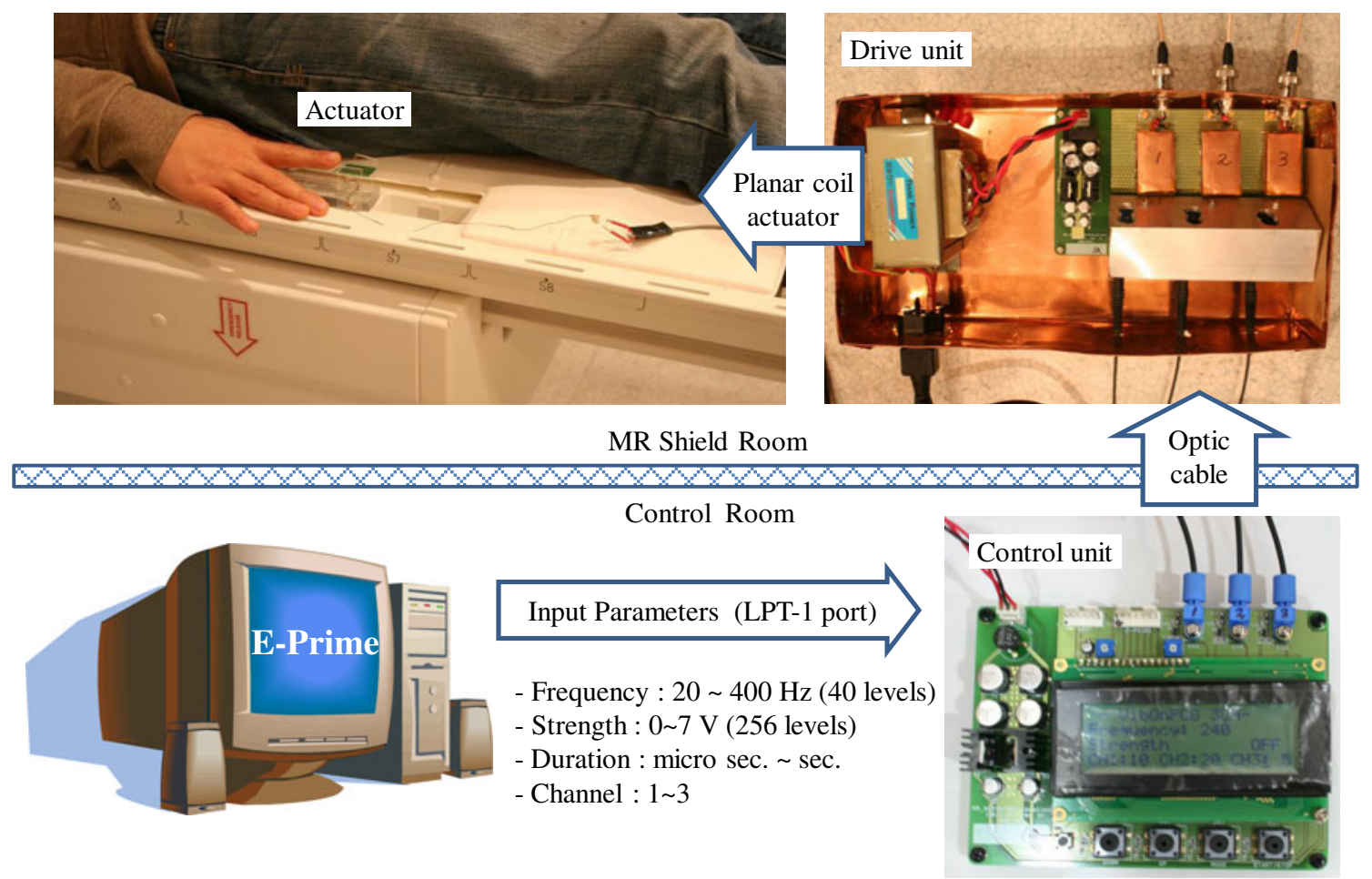

Fig. 1 Schematic of a simple magnetic-resonance-compatible vibrotactile stimulator using a planar-coil-type actuator

signals can be delivered via optic cable to the drive unit inside the MR shield room.

The drive unit operates the planar-coil-type actuator in response to commands from the control unit. Optic signals from the control unit can be converted to electric signals by a photo diode. The signals are amplified by a $20-\mathrm{W}$ audio power amplifier LM1875 (National Semiconductor, Dallas, TX). To compensate for resistance errors in the drive unit and for the resistance change due to the pattern length of the printed circuit board (PCB), a ten-turn variable resistor (R2 in Fig. 2) was used in the input port of the audio power amplifier. The amplified signals pass the filter trap and operate the planar-coil-type actuator. The filter trap can protect the drive unit from currents induced by radio frequency (RF) and the gradient pulse during the acquisition of MR images. Figure 3 shows the schematic of the filter trap, which consists of an LC resonator with an inductor and capacitor. The dimensions of drive unit are $300 \times 150 \times$ $70 \mathrm{~mm}^{3}(\mathrm{~W} \times \mathrm{L} \times \mathrm{H})$, and the weight including the power transformer is $2.6 \mathrm{~kg}$.

The planar-coil-type actuator has been developed using planar technology that constructs the planar solenoid by coiling on the PCB instead of the conventional winding of copper wire on a bobbin. The advantages of planar technology as compared with the conventional type of coil are the following: (1) excellent repeatability and thermal characteristics, (2) durability, (3) humidity resistance, (4) ability to assume various shapes, and (5) lightweight construction.

Figure 4a shows the principle of torque $(\tau)$ generation by the planar-coil-type actuator. Torque can be expressed as $\tau=$ $m \times B_{0}$, where $B_{0}$ is the strength of the static magnetic field of the MR system and $m$ is the magnetic moment, which can also be expressed as $m=I \cdot S$ ( $I$, current; $S$, area of planar coil). Since $B_{0}$ is fixed, the torque can be increased by

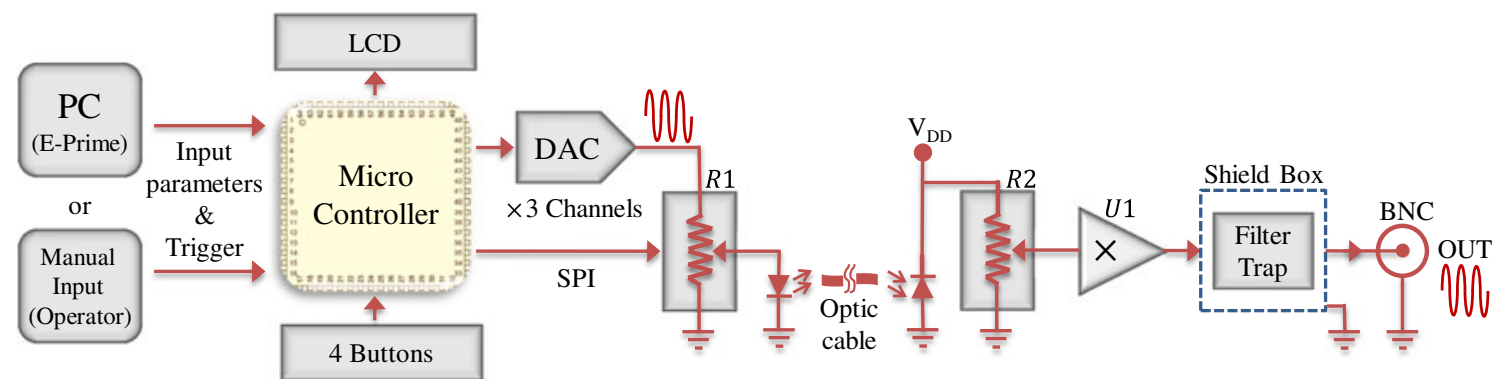

Fig. 2 Block diagram of the stimulator's control and drive units 
Fig. 3 Schematic of the filter trap
Shield Box

(Connect to GND)

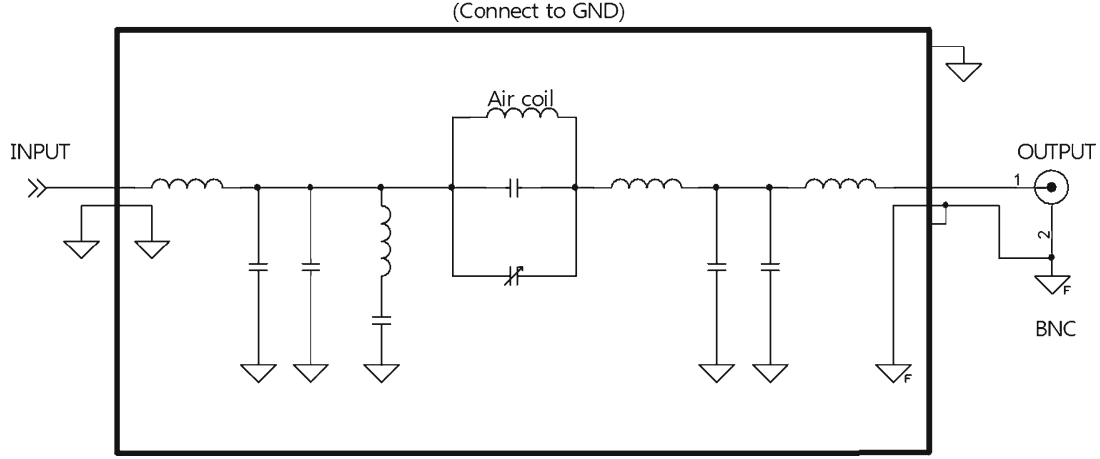

increasing either area $(S)$ or current $(I)$. The area can be controlled by the number of turns on the planar solenoid.

Figure $4 \mathrm{~b}$ shows the schematic of the planar-coil-type actuator. The extended lines equidistant around the planar-coil-type actuator can control the contact area with the skin. The planartype actuator was designed with OrCad Layout (Cadence Design Systems, Austin, TX). Figure 4c shows the actual shape of the tactile stimulator. The planar-type actuator was fixed to an acryl support with a 0.25 -mm-diameter nylon monofilament. The transient response time of the start and end of stimulation is affected by the time constant, which is determined by the inductance $(L)$ and the DC resistance $(R)$. Measuring with a low-frequency impedance analyzer (Model HP4192A, Hewlett Packard, Austin, TX), the planar-coil-type actuator has an inductance of $5 \mu \mathrm{H}$ and a DC resistance of 3.2 $\Omega$, resulting in a time constant $(L / R)$ of $1.6 \mu \mathrm{s}$. Therefore, the transient response time could be theoretically controlled on a scale of microseconds. However, the tension force of the nylon monofilament and the weight of the planar-coil-type actuator $(0.352 \mathrm{~g})$ might slow down the transient response time.

\section{Testing the MR-compatible vibrotactile stimulator using a planar-coil-type actuator}

The effect of the developed tactile stimulator on MR images was investigated. The three following conditions were compared using acquired MR images: with no planar-coiltype actuator (without an actuator); with the planar-coil-type actuator positioned $30 \mathrm{~cm}$ away from the center of the head coil but not being operated (with an actuator); and with the planar-coil-type actuator being operated in the same position (with an actuator + operation). The echoplanar cylindrical phantom images were collected with a 3.0-T MAGNETOM Trio MR scanner (Siemens Healthcare, Munich, Germany) with the following parameters: repetition time/echo time $(\mathrm{TR} / \mathrm{TE})=3,000 / 30 \mathrm{~ms}$, field of view $=192 \mathrm{~mm}$, matrix size $=128 \times 128$, slice thickness $=2 \mathrm{~mm}$, number of axial slices $=35$, and flip angle $=90^{\circ}$.

Figure 5 shows MR images from the condition in which the planar-coil-type actuator was operated (with actuator + operation). Slice B is the middle axial image, and Slices A and $\mathrm{C}$ are the axial images $26 \mathrm{~mm}$ above and below Slice B, respectively. As is shown in Fig. 5, the signal-to-noise ratio (SNR) was calculated for three cases - that is, without an actuator, with an actuator, and with an actuator + operation -by dividing each intensity of three regions - that is, Locations 1, 2, and 3-by the average intensity of four background regions (N1, N2, N3, and N4).

Table 1 shows the calculated SNRs in those three cases (without an actuator, with an actuator, and with an actuator + operation). The difference in the SNRs was very small, as is shown in Table 1, and there was no distortion of the MR images when the planar-coil-type actuator was operated, as

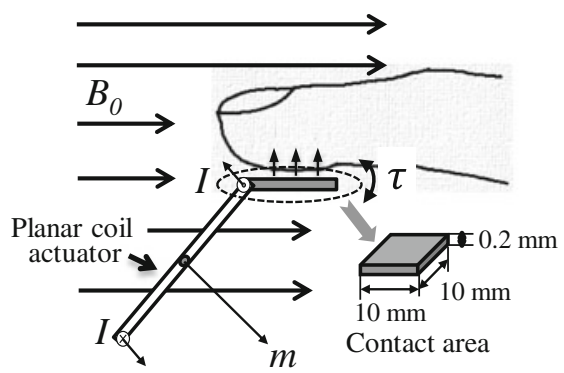

(a)

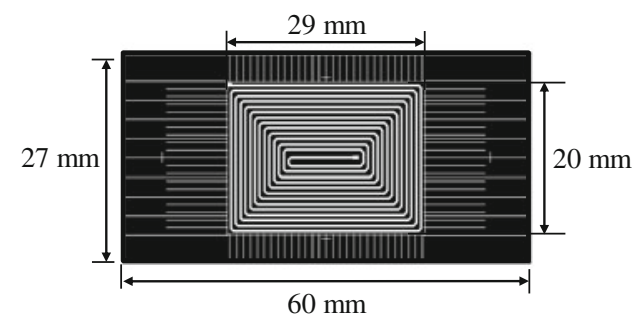

(b)

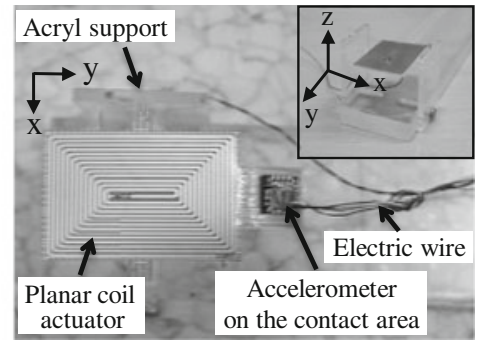

(c)
Fig. 4 a Principles behind the planar-coil-type actuator $\left(B_{0}\right.$, static magnetic field; $I$, current; $m$, magnetic moment; $\tau$, torque); $\mathbf{b}$ schematic of the planar-coil-type actuator (line width, $0.25 \mathrm{~mm}$; turns, 13; thickness, $0.2 \mathrm{~mm}$; material, fiberglass material with an epoxy resin FR-4); $\mathbf{c}$ actual shape of the planar-coil-type actuator 
Fig. 5 Magnetic resonance phantom images when the planar-coil-type actuator was operated (with an actuator + operation). Slice A, $26 \mathrm{~mm}$ above Slice B; Slice B, middle axial image; Slice C, $26 \mathrm{~mm}$ below Slice B; Locations 1, 2, and 3: regions of interest; $\mathrm{N} 1, \mathrm{~N} 2, \mathrm{~N} 3$, and $\mathrm{N} 4$ : background regions

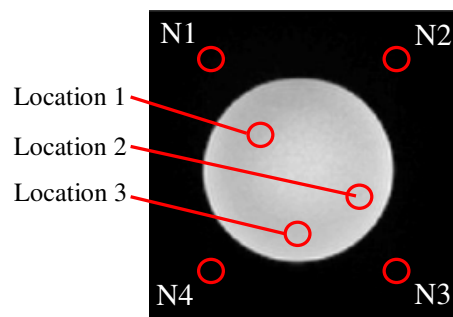

Slice A

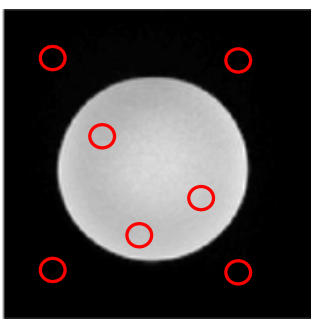

Slice B

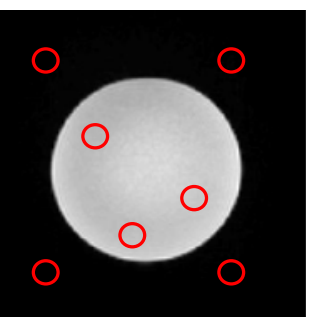

Slice C is shown in Fig. 5. Thus, we could confirm that there was no effect of the tactile stimulator that we developed on the MR images.

Figure 6 shows the performance of the filter trap in protecting the drive unit. We can protect the drive unit by attenuating the induced current from the RF and gradient pulses of the MR scanner to below $-60 \mathrm{~dB}$. As is shown in Fig. 6, the attenuated signals from input to output satisfy the condition of being below $-60 \mathrm{~dB}$ in the range from $0.3 \sim$ $150 \mathrm{MHz}$. Thus, the developed tactile stimulator can be used without any compensation for all MR scanners below $3.0 \mathrm{~T}$. The performance of the filter trap was measured with RF network analyzer 8712ET (Agilent Technologies, Santa Clara, CA).

The stable performance of the developed tactile stimulator in an MR scanner was confirmed. To measure stimulation signals from the planar-coil-type actuator, a three-axis accelerometer (Model MMA7260Q, Freescale Semiconductor, Austin, TX) was installed on the skin-contacting area (Fig. 4c). As MR images were collected (Fig. 5), acceleration (g) signals from the three-axis accelerometer were measured with a TDS2014 (Tektronix, Beaverton, OR) four-channel digital oscilloscope (sampling rate 2,500 samples/s) in ACcoupling mode. Figure 7 a shows the output of the three-axis accelerometer with a $250-\mathrm{Hz}$ stimulation frequency. Since the static magnetic field is in the direction of the $y$-axis and current is in the direction of the $x$-axis, the maximum force is induced in the direction of the $z$-axis, which is orthogonal to the $x-y$ plane. To investigate the stimulation intensity changes due to frequency changes, the peak values in the $z$-axis direction of the three-axis accelerometer were measured at five different stimulation intensities (Strengths 1, 5, 10, 15, and 20) with varying stimulation frequencies $(20 \sim 400 \mathrm{~Hz})$. As is shown in
Fig. 7b, the various stimulation intensities are almost constant with changing stimulation frequencies. As is evident from the results of the power spectral density analysis, an accurate stimulation frequency could be generated, since frequency components other than the dominant frequency component were negligible (Fig. 7c).

\section{fMRI experiment using the developed stimulator}

Preliminary fMRI experiments were performed to test the applicability of the developed stimulator. Three male subjects $(26.3 \pm 1.5$ years old $)$ were treated with three stimulation frequencies $(100,250$, and $400 \mathrm{~Hz})$ and stimulation intensities (3, 8, and 13 levels), and fMRI data were obtained. We used the same parameters as in the phantom image experiments. The experiment consisted of nine trials of the vibrotactile stimulation testing. Each trial consisted of two blocks; each block had both stimulation (30 s) and resting (30 s) phases. In stimulation phase, nine different stimulations (three frequencies and three intensities) were applied on the distal phalanx of the right index finger in a random order, and no stimulation was applied in the resting phase. All subjects closed their eyes and wore a headset to prevent disturbances from the surroundings. The fMRI data were analyzed with SPM 8 (Wellcome Department of Cognitive Neurology, London, U.K.). All functional images were aligned with the anatomic images of the study by using affine transformation routines built into SPM 8. The realigned scans were coregistered to the participant's anatomic images obtained within each session and were normalized to SPM8's template image. Motion correction was done using sync interpolation. The time-series data were filtered with a

Table 1 Signal-to-noise ratios of the magnetic resonance image in three conditions (i.e., without an actuator, with an actuator, and with an actuator + operation) for three slices (A, B, and C) at three regions (L1, L2, and L3)

\begin{tabular}{|c|c|c|c|c|c|c|c|c|c|c|c|c|}
\hline & \multicolumn{4}{|l|}{ Slice A } & \multicolumn{4}{|l|}{ Slice B } & \multicolumn{4}{|l|}{ Slice C } \\
\hline & L1 & L2 & L3 & Avg. & $\mathrm{L} 1$ & $\mathrm{~L} 2$ & L3 & Avg. & L1 & L2 & L3 & Avg. \\
\hline Without an actuator & 182.04 & 209.32 & 196.68 & 196.01 & 194.00 & 218.40 & 206.04 & 206.15 & 184.80 & 214.80 & 204.24 & 201.28 \\
\hline With an actuator & 185.04 & 211.76 & 197.72 & 198.17 & 193.96 & 220.32 & 207.00 & 207.09 & 182.48 & 211.00 & 205.08 & 199.52 \\
\hline $\begin{array}{l}\text { With an actuator }+ \\
\text { operation }\end{array}$ & 185.08 & 212.28 & 200.64 & 199.33 & 194.16 & 219.84 & 206.00 & 206.67 & 186.20 & 212.44 & 203.80 & 200.81 \\
\hline
\end{tabular}


Fig. 6 Performance of the filter trap

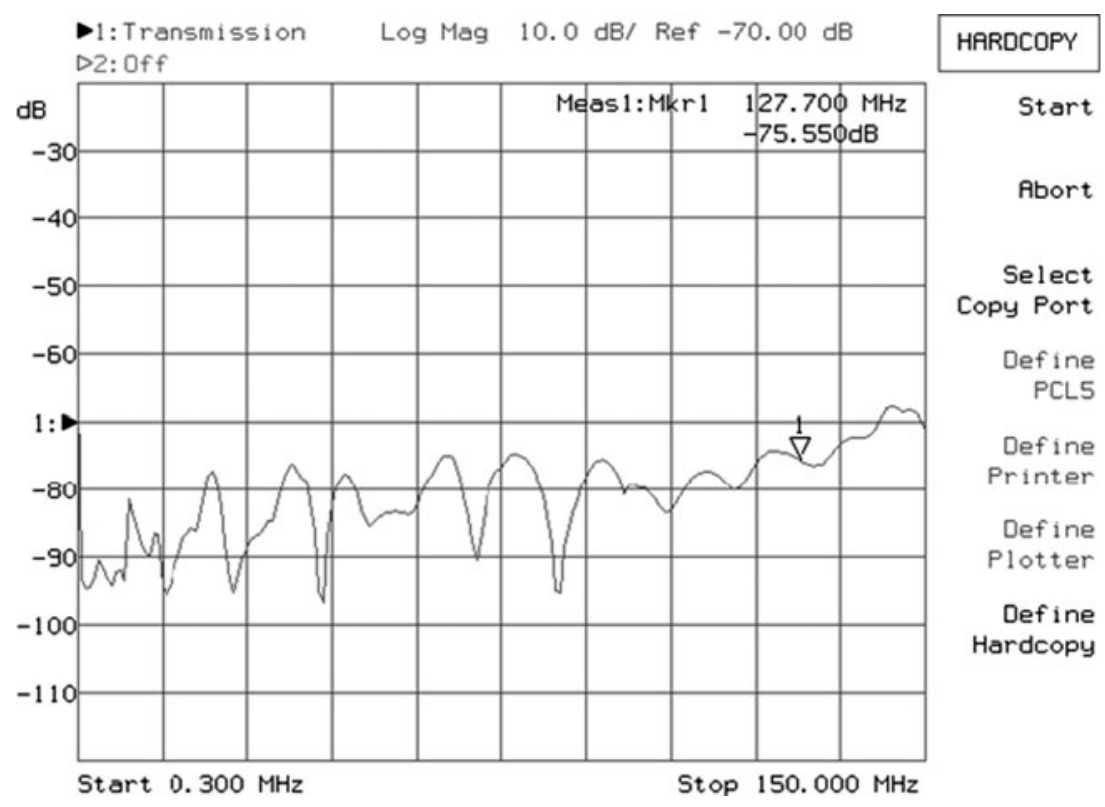

180-s high-pass filter to remove artifacts due to cardiorespiratory and other cyclical influences. The functional map was smoothened with a 7-mm isotropic Gaussian kernel prior to statistical analysis. The statistical analysis was done as a group using the general linear model and the theory of Gaussian random fields implemented in SPM 8. Using the subtraction procedure, activated areas between the stimulation phase and the resting phase were color-coded using $t$ scores.

The results of the fMRI data showed that the activation region of the somatosensory area increased as the stimulation frequency increased at a fixed stimulation intensity. The activation region of postcentral gyrus (BA 3) increased as stimulation intensity increased at the $100-$ and $250-\mathrm{Hz}$ stimulation frequencies, while the activation region of postcentral gyrus (BA 5) increased as stimulation intensity increased at the $400-\mathrm{Hz}$ stimulation frequency (Fig. 8).

\section{Discussion}

In this study, we developed a simple MR-compatible planarcoil-type actuator to generate vibrotactile stimulation under the static magnetic field of an MR system. The developed tactile stimulator was stable in the MR environment and showed no distortion of MR images. With fMRI, we observed brain activation changes from both stimulation frequency and intensity changes.

The new tactile stimulator has many advantages over the conventional pneumatic, piezoelectric-element, shaft, and electromagnetic stimulators in an MR environment.

With respect to the system components, this tactile stimulator consists of three parts: a control unit, drive unit, and planar-coil actuator. Our system is small, simple, and inexpensive, with a wide variety of applications. The new

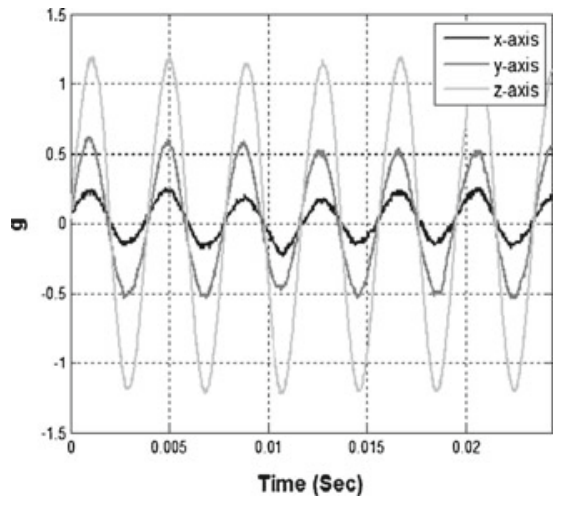

(a)

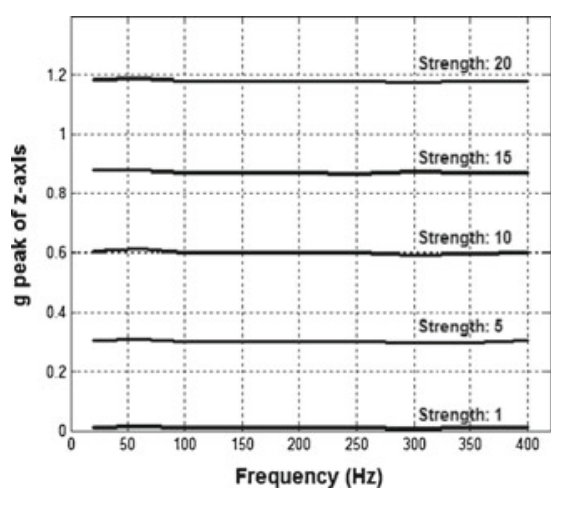

(b)
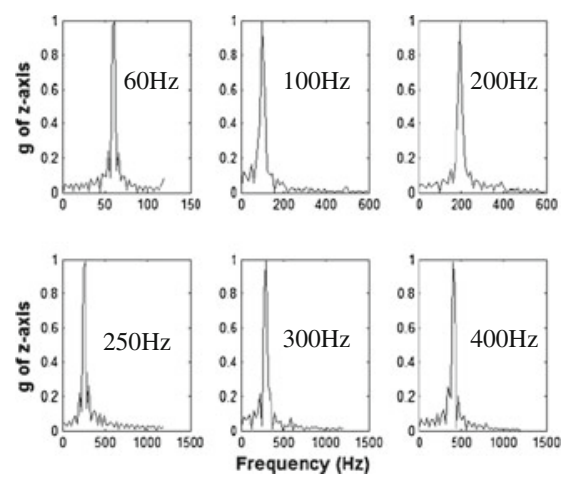

(c)
Fig. 7 Response characteristics of the planar-coil-type actuator during acquisition of magnetic resonance images. a Acceleration (g) signals of a three-axis accelerometer at the $250-\mathrm{Hz}$ stimulation frequency; b $\mathrm{g}$ peak of the $z$-axis at five different stimulation intensities (Strengths 1,
$5,10,15$, and 20$)$ with varying stimulation frequencies $(20-400 \mathrm{~Hz})$; c power spectral density with six different stimulation frequencies $(60$, $100,200,250,300$, and $400 \mathrm{~Hz}$ ) 


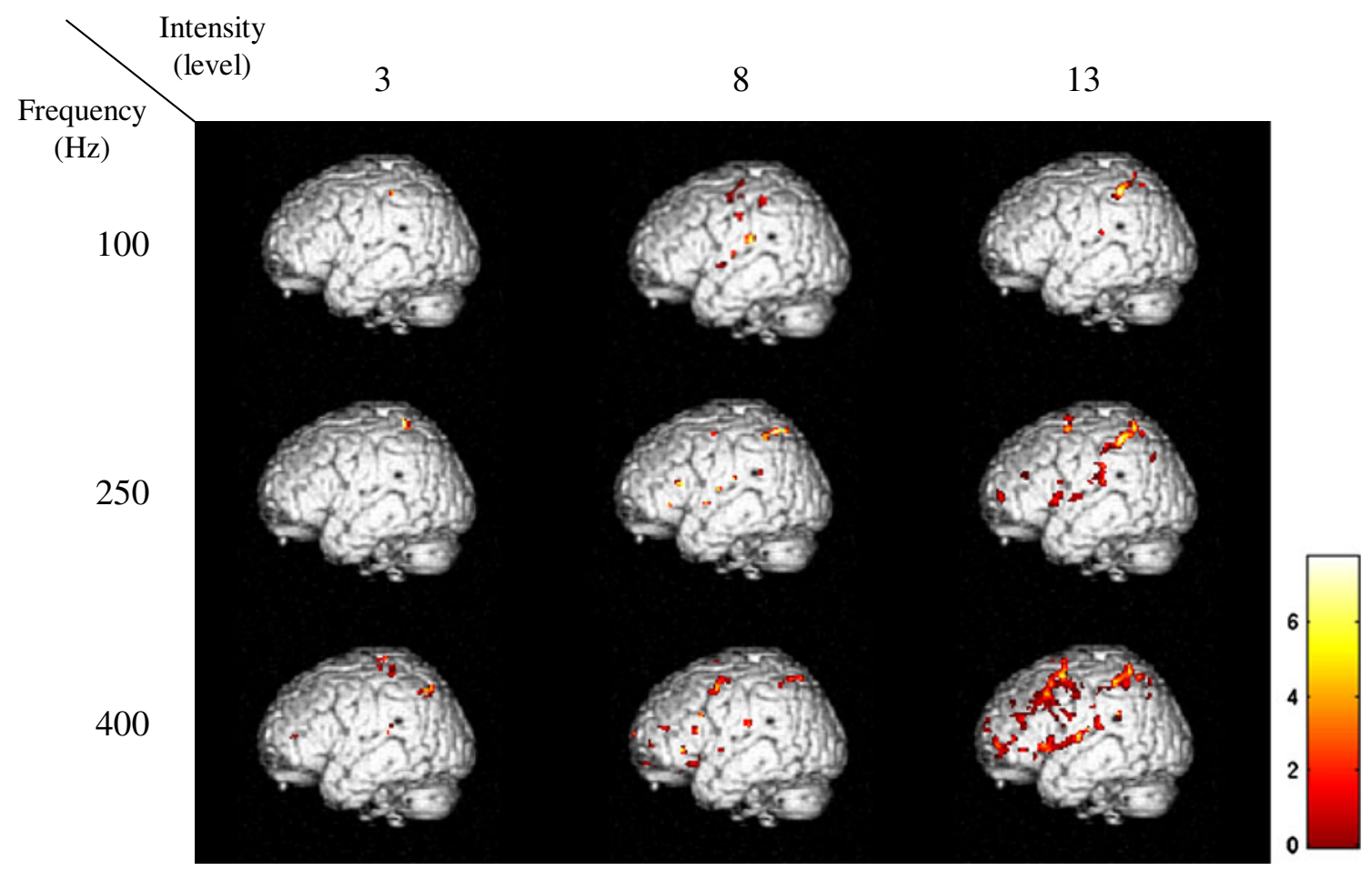

Fig. 8 Brain activation changes at different stimulation frequencies (100, 250, and $400 \mathrm{~Hz})$ and stimulation intensities (3, 8, and 13 levels)

stimulator can be operated manually or with a PC. In particular, since the stimulator can be controlled with E-Prime software, tactile, auditory, and visual stimulation could be generated simultaneously. With the excellent performance of the filter trap in protecting the drive unit, the developed stimulator can be used without compensation with all MR scanners below 3.0 T.

With respect to stimulation, the new stimulator has a wide range of stimulation frequencies $(20-400 \mathrm{~Hz}$, at 40 levels) and stimulation intensities (0-7 V, at 256 levels). Also, stimulation intensity does not change due to frequency change. With a fast transient response time (ideally, $1.6 \mu \mathrm{s}$ ), stimulation time can be controlled on a scale of microseconds. Moreover, sinusoidal, square, triangular, saw-tooth, and arbitrary waves can be generated by changing the binary code values.

With respect to the actuator, it delivers a repeatable signal, is durable, is stable at various temperatures, and allows for various shapes of the coiling on the printed circuit board. Also, the contact area between the skin and the tactile actuator can easily be adjustable.

Even though it was possible to stimulate below $20 \mathrm{~Hz}$ with the developed system, we could not get the precise sinusoidal wave form in this frequency region, and second and third harmonic elements were shown in the analysis of power spectral density. Thus, we limited the stimulation frequency range from 20 to $400 \mathrm{~Hz}$. Similar to other vibrotactile stimulation methods above $20 \mathrm{~Hz}$, our system has limitations in controlling the contact force. These limitations should be overcome in the future.

Since the new tactile stimulator uses the static magnetic field $\left(B_{0}\right)$ of an MR scanner, it has two limitations. First, intensity can be changed with the position and direction of the planarcoil-type actuator (Graham et al., 2001). However, human subjects usually do not move during tactile fMRI studies, and the position of the planar-coil-type actuator can be fixed to provide a consistent stimulation intensity. Second, acquiring sufficient stimulation on the lower leg, including the foot, is not easy, because of the weak static magnetic field around that part of the body. However, other body sites can be stimulated with various shapes of actuator and multiple channels, because the planar coil can be constructed in a number of ways.

Brain activation changes were observed using the developed system with a range of stimulation frequencies (100, 250, and $400 \mathrm{~Hz}$ ) and intensities (3, 8, and 13 levels). In previous work (Hegner, Lee, Grodd, \& Braun, 2010), differences in blood oxygen level dependent (BOLD) intensity were extracted in the low stimulation frequency range (16 $32 \mathrm{~Hz}$ ). As stimulation frequency increased, BOLD intensity increased. The activation region of the somatosensory area increased as stimulation intensity increased with a fixed stimulation frequency (Graham et al., 2001). Even though the range of stimulation frequencies was different, our results showed, similarly, that the activation region of the somatosensory area increased with increasing stimulation frequencies and intensities These results imply that the 
developed stimulator can be used for fMRI studies with a wide range of stimulation frequencies and intensities.

The tactile stimulator we have developed has many advantages in terms of its systemic, stimulation, and actuator characteristics over conventional tactile stimulators. Therefore, the new tactile stimulator can be used in neuroscience and behavioral science for quantitative study of the characteristics of tactile cognition.

Author note This research was supported by the Pioneer Research Center Program through the National Research Foundation of Korea funded by the Ministry of Education, Science and Technology (Grant No. 2011-0027920). This work was also supported by the National Research Foundation of Korea (NRF) grant funded by the Korea government (MEST) (Grant No. 2012-007998).

\section{References}

Anton, J. L., Benali, H., Guigon, E., Di-Paola, M., Bittoun, J., Jolivet, O., \& Burnod, Y. (1996). Functional MR imaging of the human sensorimotor cortex during haptic discrimination. NeuroReport, 7 , 2849-2852.

Bleton, J. P., Vidailhet, M., Bourdain, F., Ducorps, A., Schwartz, D., Delmaire, C., . . Meunier, S. (2011). Somatosensory cortical remodelling after rehabilitation and clinical benefit of in writer's cramp. Journal of Neurology, Neurosurgery and Psychiatry, 82, 574-577.

Briggs, R. W., Fy-Liacco, I., Malcolm, M. P., Lee, H., Peck, K. K., Gopinath, K. S., . . . Tran-Son-Tay, R. (2004). A pneumatic vibrotactile stimulation device for fMRI. Magnetic Resonance in Medicine, 51, 640-643.

Chakravarty, M. M., Broadbent, S., Rosa-Neto, P., Lambert, C. M., \& Collins, D. L. (2009). Design, construction, and validation of an MRI-compatible vibrotactile stimulator intended for clinical use. Journal of Neuroscience Methods, 184, 129-135.

Gallace, A., Tan, H. Z., Haggard, P., \& Spence, C. (2007). Short term memory for tactile stimuli. Brain Research, 1190, 132-142.

Gallasch, E., Fend, M., Rafolt, D., Nardone, R., Kunz, A., Kronbichler, M., . . . Golaszewski, S. (2010). Cuff-type pneumatic stimulator for studying somatosensory evoked responses with fMRI. Neurolmage, 50, 1067-1073.

Geyer, S., Schleicher, A., Schormann, T., Mohlberg, H., Bodegard, A., Roland, P. E., \& Zilles, K. (2001). Integration of microstructural and functional aspects of human somatosensory areas $3 \mathrm{a}, 3 \mathrm{~b}$, and 1 on the basis of a computerized brain atlas. Journal of Anatomy and Embryology, 204, 351-366.

Gizewski, E. R., Koeze, O., Uffmann, K., de-Greiff, A., Ladd, M. E., \& Forsting, M. (2005). Cerebral activation using a MR-compatible piezoelectric actuator with adjustable vibration frequencies and in vivo wave propagation control. NeuroImage, 24, 723-730.

Golaszewski, S. M., Siedentopf, C. M., Baldauf, E., Koppelstaetter, F., Eisner, W., Unterrainer, J., . . . Felber, S. R. (2002). Functional magnetic resonance imaging of the human sensorimotor cortex using a novel vibrotactile stimulator. NeuroImage, 17, 421-430.

Graham, S. J., Staines, W. R., Nelson, A., Plewes, D. B., \& McIlroy, W. E. (2001). New devices to deliver somatosensory stimuli during functional MRI. Magnetic Resonance in Medicine, 46, 436-442.

Harrington, G. S., Wright, C. T., \& Downs, J. H. (2000). A new vibrotactile stimulator for functional MRI. Human Brain Mapping, 10, 140-145.

Hegner, Y. L., Lee, Y., Grodd, W., \& Braun, C. (2010). Comparing tactile pattern and vibrotactile frequency discrimination: A human fMRI study. Journal of Neurophysiology, 103, 3115-3122.

Ho, C., \& Spence, C. (2007). Head orientation biases tactile localization. Brain Research, 1144, 236-241.

Kim, H. S., Yeon, H. W., Choi, M. H., Kim, J. H., Choi, J. S., Park, J. Y., . . Chung, S. C. (2011). Development of a tactile stimulator with simultaneous visual and auditory stimulation using E-Prime software. Computer Methods in Biomechanics and Biomedical Engineering. doi:10.1080/10255842.2011.625018

Lynette, A. J., \& Nadine, B. S. (2008). Tactile displays: Guidance for their design and application. Human Factors, 50, 90-111.

Montant, M., Romaiquere, P., \& Roll, J. O. (2009). A new vibrator to stimulate muscle proprioceptors in fMRI. Human Brain Mapping, 30, 990-997.

Roiha, K., Kiveskari, E., Kaste, M., Mustanoja, S., Makela, J. P., Salonen, O., . . . Forss, N. (2011). Reorganization of the primary somatosensory cortex during stroke recovery. Clinical Neurophysiology, 122, 339-345.

Tsekos, N. V., Khanicheh, A., Christoforou, E., \& Mavroidis, E. (2007). Magnetic resonance-compatible robotic and mechatronics systems for image-guided interventions and rehabilitation: A review study. Annual Review of Biomedical Engineering, 9, 351-387.

Wienbruch, C., Candia, V., Svensson, J., Kleiser, R., \& Kollias, S. S. (2006). A portable and low-cost fMRI compatible pneumatic system for the investigation of the somatosensory system in clinical and research environments. Neuroscience Letters, 398, 183-188. 